\title{
Assessing Short Term Change in Carpal Bone Age Assessments by Dual-Energy X-Ray Absorptiometry
}

\author{
Jingmei Wang ${ }^{1}$, Yun Sun ${ }^{2}$ and Tom V Sanchez ${ }^{3 *}$ \\ ${ }^{1}$ Research and Development, Norland at Swissray, Beijing, China \\ ${ }^{2}$ Department of Radiology, Hospital of Tsinghua University, Beijing, China \\ ${ }^{3}$ Research and Development, Norland at Swissray, Socorro, NM, USA
}

*Corresponding author: Tom V Sanchez, Research and Development, Norland at Swissray, PO Box 1476, Socorro, NM 87801, USA, Tel: 1-505-440-9913, Fax: 1-575-838-0167, E-mail: sanchez_tom@hotmail.com

\begin{abstract}
Bone age assessments are generally done using radiography of the hand and wrist to compare the child's maturity to known standards. While a valuable study, a traditional bone age study is not very reflective of short term change. DXA is proposed as able to more precisely reflect both absolute assessments and short-term changes in maturation. The current study compares radiographic with Norland bone age assessments to compare studies and sensitivity to change in boys and girls.
\end{abstract}

Four subjects (between 9 and 16-years-old) underwent three repeated studies of DXA-based bone age assessments to assess precision. Eighteen males subjects (12 to 19-years-old) and twenty female subjects (12 to 16-yearsold) underwent radiographic (GE Discovery XR656 Digital Radiography System) and DXA (Norland XR-800) based bone age assessments to assess agreement between methods. To assess sensitivity to change in the DXA based study, 34 males (6 to 19-years-old) and 37 females (6 to 16-years-old) underwent two DXA-based bone age assessments with three months between studies to assess if a significant increase had occurred in bone mineral. Agreement in age assessment by radiographic and DXA-based bone age study was by regression analysis while assessment of sensitivity to a three-month change in DXA-based assessments was established if follow-up results showed an increase greater than the least significant change limit.

Repeatability from three measurements on four subjects showed repeatability of $1.35 \%$ (with a computed Least Significant Change Limit of $3.78 \%$ ) could be expected from the study. X-ray and DXA assessed bone age were found to be highly correlated in males (DXA Bone Age $=14.5777+$ $0.9287 x ; r=0.9897 ; P<0.001$ ) and females (DXA Bone Age $=41.2652+0.7619 x ; r=0.9250 ; p<0.001$ ) suggesting that the two studies are showing similar gross response to bone age. Seventeen of the 33 male subjects showed increases greater than the $3.78 \%$ Least Significant Change Limit--the remaining 16 subjects showed differences under $3.78 \%$ for the two studies. Examining 34 female subjects, 16 subjects showed increases greater than the $3.78 \%$ Least Significant Change Limit--the remaining 18 subjects showed differences below $3.78 \%$ in the two studies.

The study demonstrates that the Norland DXA system fitted with Bone Age Assessment Software reflects bone age similarly to that achieved by radiography and can identify significant change in children in periods as short as three months. We conclude that Bone Age Assessment Software can be a useful bone age assessment and can complement traditional radiographic studies when assessment of shortterm change in bone age is desired.

\section{Keywords}

Dual-energy x-ray absorptiometry, Skeletal age assessment, Bone age

\section{Abbreviations \\ DXA: Dual Energy X-Ray Absorptiometry; LSCL: Least Sig- nificant Change Limit}

\section{Introduction}

The bone age or skeletal age assessment is a clinically useful methodology when evaluating pediatric subjects with growth disorders. Most commonly these assessments are performed by trained radiologists comparing the patient hand and wrist radiograph-sometimes from trained visual assessment and sometimes from specialized computer assessment to radiographs from a reference database [1]. These assessments are espe- 


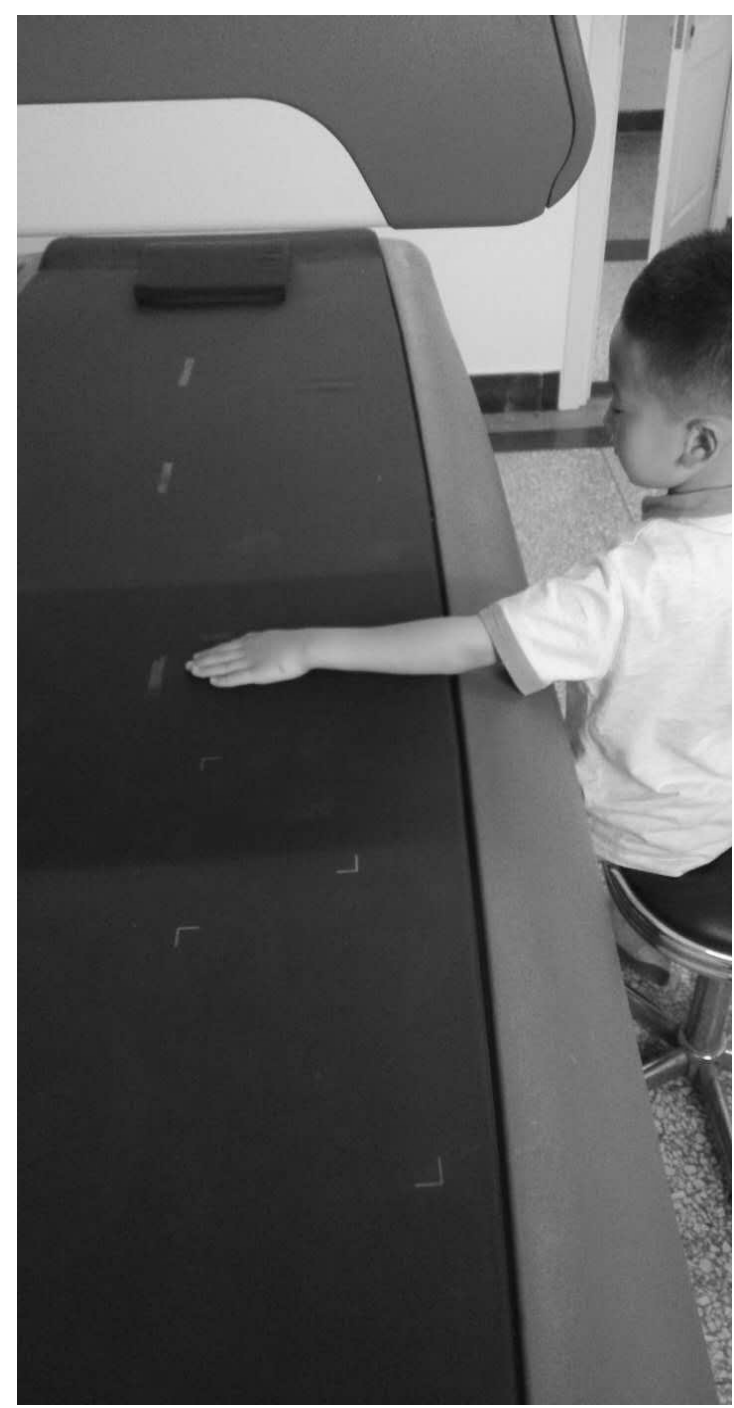

Figure 1: Subjects were positioned at the scanner with their hand laying flat in the scan region defined by the operator.

cially useful as a single evaluation or as an assessment of change over time when diagnosing and managing endocrine disorders, evaluating metabolic and growth abnormalities or delayed maturation as a result of different syndromes. Because bone age assessments are based on a radiographic study, these studies depend on changes in both mineral content in bone and on structural changes in bone. As such, assessing change with reliability can easily take a year or more between studies. Because DXA-based bone age assessments are based on quantitative assessments of bone mineral content relatively free of the more subjective estimate provided by the trained eye completing the traditional radiographic bone age study, the DXA-based bone age assessment has been proposed as a sensitive alternative bone age study [2].

\section{Methods}

The study consisted of three projects evaluating the DXA-based bone age assessment-an evaluation of repeatabiltiy, an evaluation of agreement with traditional radiographic bone age and a reflectiveness of short term change. DXA-based bone age studies were

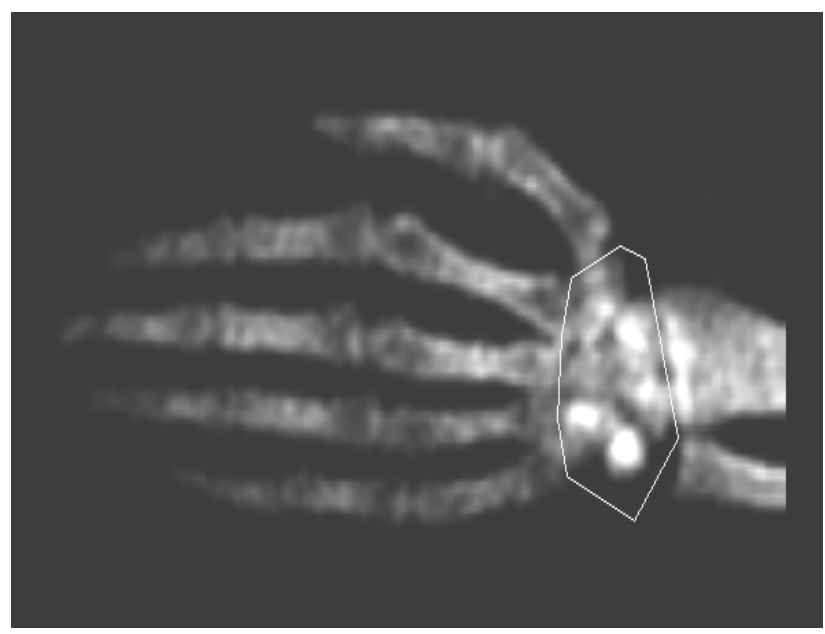

Figure 2: Image shows a DXA study of the hand from a thirteen-year-old body with the eight-sided region of interest placed to include the carpal bones. The final analysis will provide an assessment of total carpal bone mineral density.

performed using a Norland XR-800 (Ft Atkinson, WI, USA) fitted with the Research Scan Module. Subjects were the children of the facility staff and accompanied by their parent who gave consent and answered a short clinical history to rule out disorders that might compromise bone age. Subjects were evaluated with their hand laying flat on the tabletop in a region defined by the operator (Figure 1). Measurement scans were completed with settings for resolution of $1.0 \times 1.0 \mathrm{~mm}$ and with a scan speed of $90 \mathrm{~mm} / \mathrm{s}$ with analysis being carried out applying an eight-sided region of interest capturing the carpal bone mineral density (Figure 2). Studies of the hand with these setting were estimated to generate a skin entrance radiation dose of approximately $1.35 \mu \mathrm{Sv}$.

The first task in this study sought to evaluate study repeatability. Four children ( 9 to 16 years of age) underwent three repeated bone age studies with repositioning to assess repeatability between studies. Analysis proceeded with the results from the three studies on a single individual being averaged to compute a coefficient of variation.

Coefficient of variation for the four subjects were then averaged to produce a value for repeatability on the study to allow computation of the Least Significant Change Limit (LSCL).

The second task in this study sought to evaluate agreement between the DXA-based bone age study and traditional radiographic bone age. Eighteen male subjects (12-19-years-old) and twenty female subjects (12 to 16-years-old) underwent a modified Greulich and Pyle radiographic bone age assessment using the GE Discovery XR656 Digital Radiography System (GE Healthcare, Buckinghamshire, UK) and DXA-based bone age assessment using the Norland XR-800. Greulich and Pyle radiographic bone age was assigned by a trained radiologist (SY) and DXA-based bone age was assigned to subjects by a trained DXA operator (WJM) using Norland reference values [3]. The relationship between bone age assessed radiographi- 


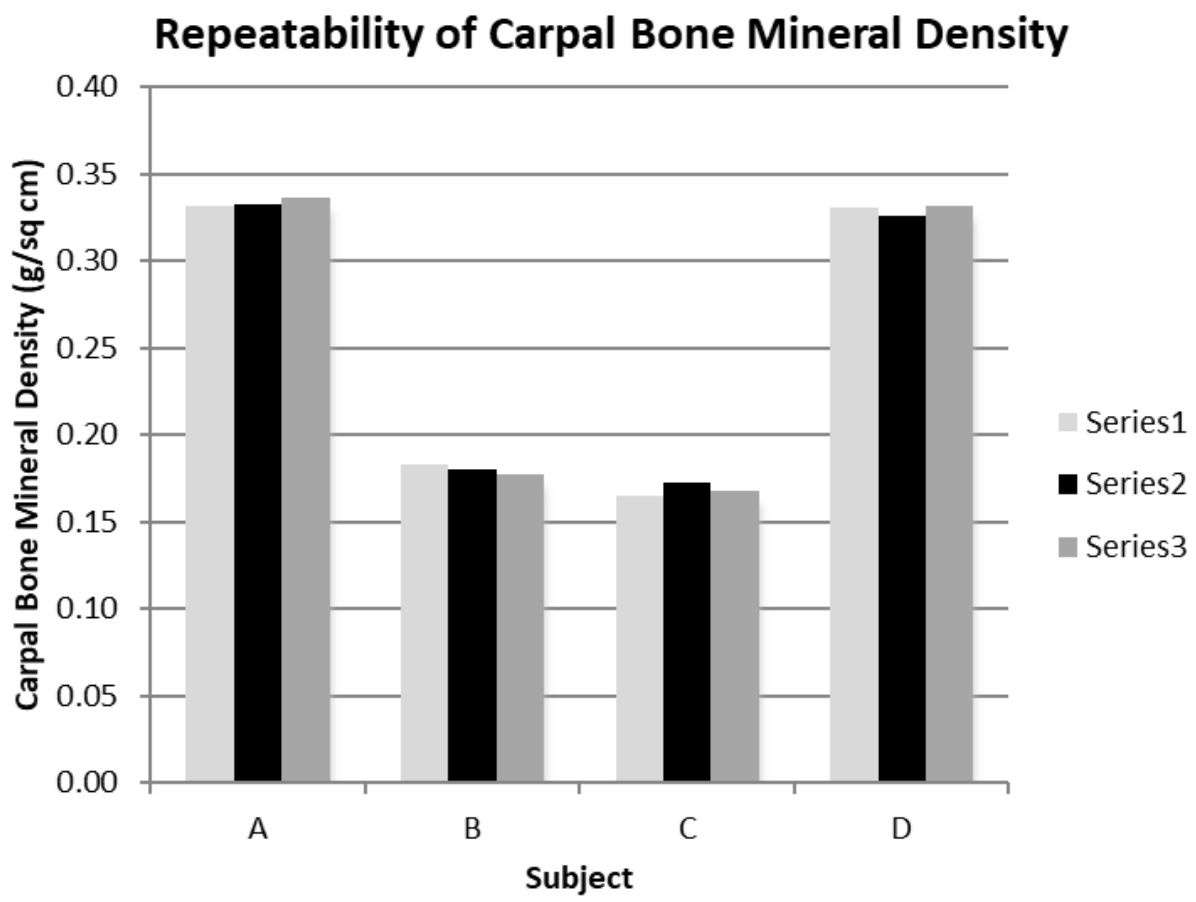

Figure 3: Repeatability of carpal bone mineral density $\left(\mathrm{gm} / \mathrm{cm}^{2}\right)$ in four subjects between 9 and 16 years of age with three repeated assessments. Average repeatability of a carpal bone density on the three studies performed on the four subjects was $1.35 \%$ which computes to a Least Significant Change Limit of $3.78 \%$.

\section{Relationship between X-ray and DXA-based Bone Age in Male Subjects}

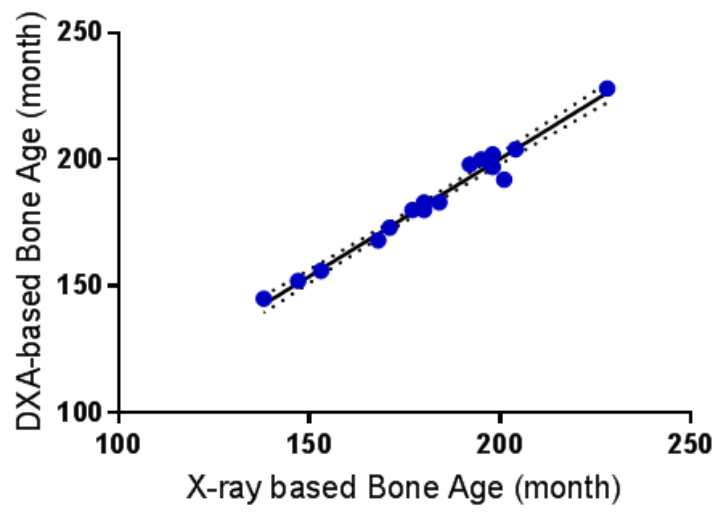

\section{Relationship between X-ray and DXA-based Bone Age in Female Subjects}

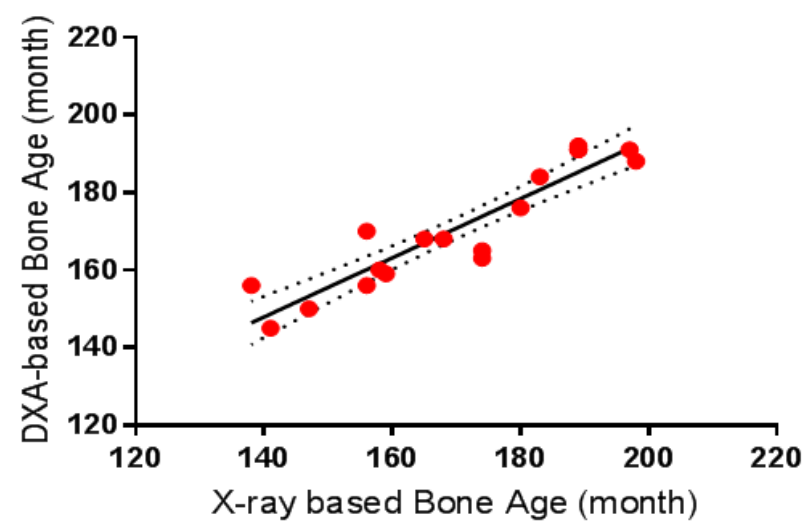

Figure 4: Relationship between traditional radiographically and DXA-based bone age assessment in male and female subjects. Strong positive regressions $(Y=0.9288 X+14.58 ;$ Sy. $x=3.295 ; r=0.9897)$ and $(Y=0.7619 X+41.27 ;$ Sy $. x=5.727 ; r=0.9249)$ with little variation about the regression lines are seen with male and female subjects, respectively.

cally and by DXA was evaluated by regression analysis and by a Bland-Altman assessment.

The third task in this study sought to evaluate the sensitivity to short-term change in DXA based bone age studies in normal children. Two DXA-based bone age studies were performed on 34 males (6-19 years old) and 37 females (6-16 years-old) with three months between the studies to assess if a significant change-defined as a change greater than the computed LSCL-was reflected by the DXA-based bone age study.

\section{Results}

Four children, including three females ( 9 to 16 -yearsold) and one male (15-years-old), were evaluated three times each showing an average DXA-based carpal bone mineral density between $0.1685 \mathrm{gm} / \mathrm{cm}^{2}$ and 0.3337 $\mathrm{gm} / \mathrm{cm}^{2}$ (Figure 3). Assessing repeatability of bone age in these studies shows a repeatability calculated as a coefficient of variation of $1.35 \%$ on bone mineral density (approximately 2.2 months-on bone age for these four subjects)-which results in a computed LSCL of $3.78 \%$.

Examining the relationship between traditional radiographic and DXA-based bone age assessments show strong positive regressions in a population of eighteen male $(y=$ $0.9288 x+14.58 ;$ Sy. $x=3.295 ; r=0.9897)$ and twenty female $(y=0.7619 X+41.27 ; S y . x=5.727 ; r=0.9249)$ subjects (Figure 4). Given that there seemed to be little variation about the regression lines a Bland-Altman evaluation was 


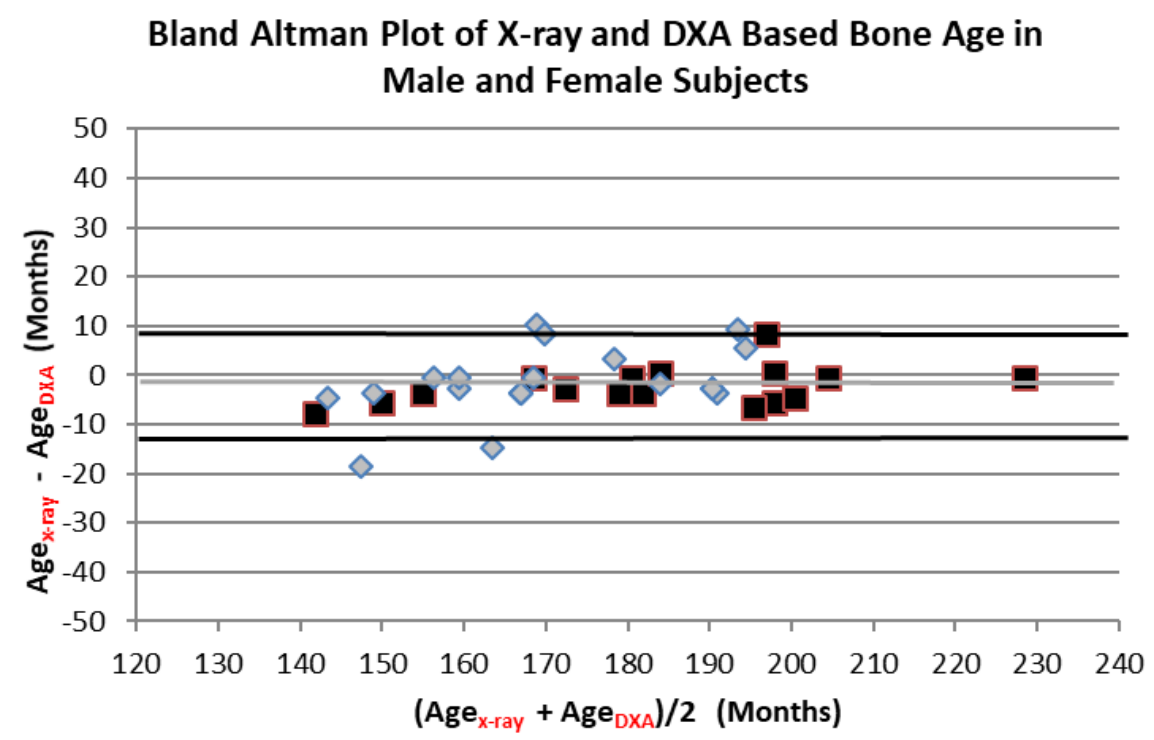

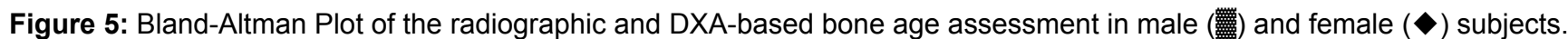
A Bland-Altman Plot shows a Mean Value of -1.34 months with an upper Limit of Agreement at 9.64 and a lower Limit of Agreement at -12.32 months.

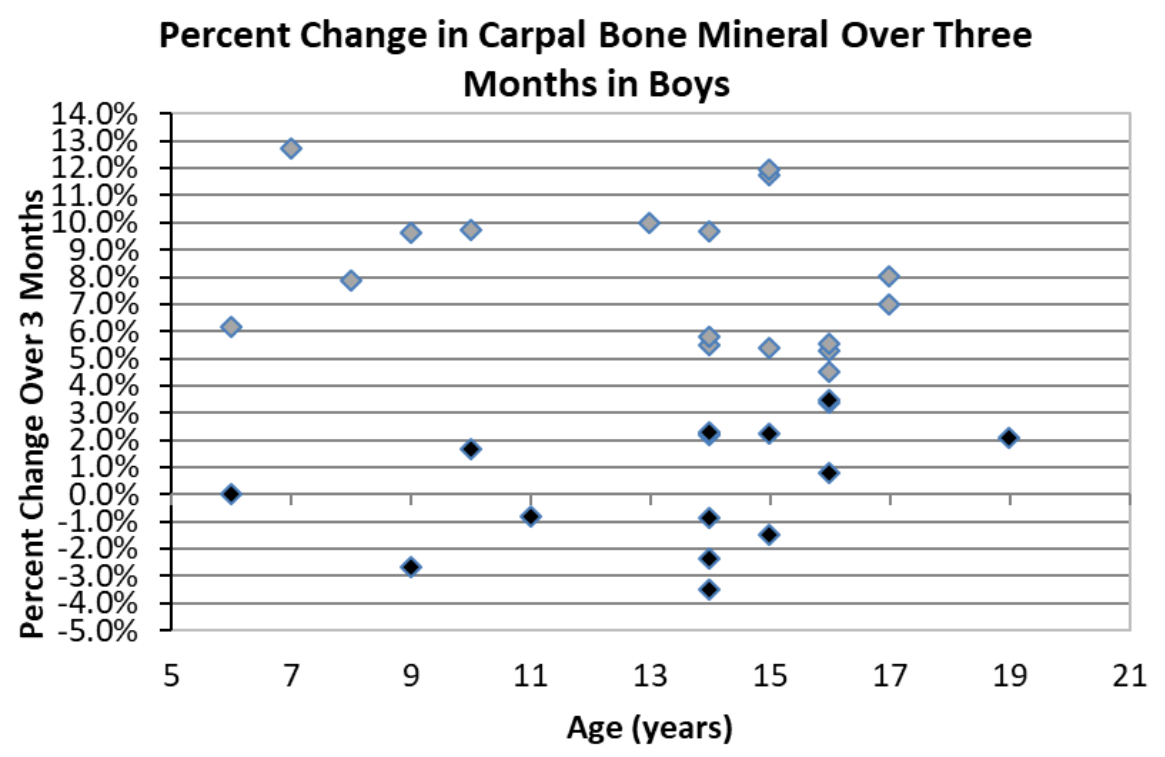

Figure 6: Examining the change in carpal bone density in 33 boys between 6 and 19-years-old showed a significant--exceeding the LSCL threshold of 3.78\%-three month increase in $17(\diamond)$ boys.

conducted to assess the potential to consider the DXAbased bone age assessment as a stand-alone bone age assessment (Figure 5). Processing the radiographic and DXAbased bone age from the 38 children in a Bland-Altman Plot shows a mean radiographic to DXA-based difference of -1.34 months with an upper Limit of Agreement of 9.64 months and a lower Limit of Agreement of -12.32 months. Taken together the regression studies and the Bland-Altman assessment would suggest that while radiographic and DXA-based bone age assessments follow each other well the radiographic assessment may be better relied upon in the single clinical assessment.

Examining the sensitivity to short-term change the study compared two carpal bone mineral densities taken three months apart in 33 boys ( $6-19$ years old) and 37 girls (6-16 years old). A significant change was noted if the difference between the two studies were found to exceed the computed LSCL. The study showed that 17 boys between 6-19 years old reflected three-month increases exceeding the LSCL threshold of $3.78 \%$ (Figure 6). The remaining 16 boys (48\%) showed values that stayed within the $3.78 \%$ threshold suggesting that there was neither a significant increase or decrease in the three-month period. The study also showed that 16 girls between $6-16$ years old reflected three-month increases exceeding the LSCL threshold of $3.78 \%$ (Figure 7). The remaining 18 girls (53\%) showed values that stayed within the $3.78 \%$ threshold suggesting that there was neither a significant increase or decrease in the three-month period. Examination of the data also revealed that significant increases were seen throughout the age range examined in both the boys and girls.

\section{Discussion}

Bone age assessment is clinically important in the as- 


\section{Percent Change in Carpal Bone Mineral Over Three Months in} Girls

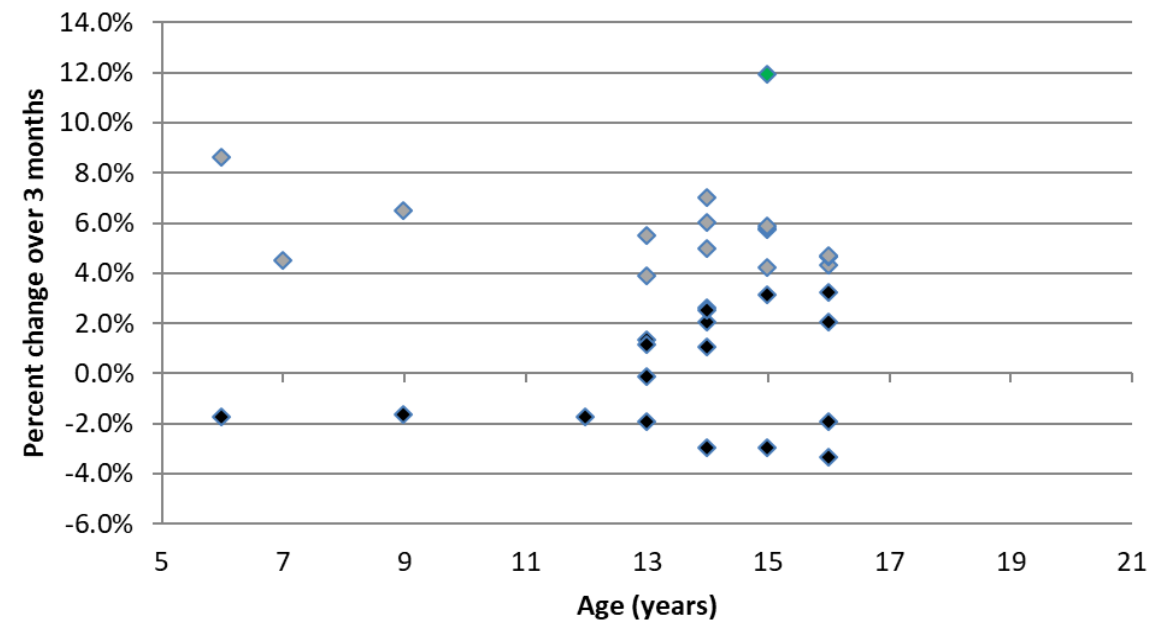

Figure 7: Examining the change in carpal bone density in 34 girls between 6 and 16-years-old showed a significant-exceeding the LSCL threshold of $3.78 \%$-three month increase in $16(\diamond)$ girls.

sessment of disease and in evaluating the response to treatment. Critical applications of bone age assessment can be found in wide-ranging setting going from endocrinopathies to planning for orthopedic procedures. While a number of technologies are used in bone age assessment the most widely applied are the Greulich and Pyle Method or the Tanner-Whitehouse Method based on traditional radiographic readings [4]. While invaluable, given the reliance on making an assessment based on both content and structure, these radiographically based bone age assessments can be difficult to execute with consistency. Indeed, as noted by Martin and associates, reports in the literature suggest that when evaluated on repeated assessments standard errors in these bone age assessments may range from 5.4 months to 9.8 months. In an effort to improve consistency and efficiency, computer-based bone age assessment has been proposed as a methodology. Computer based bone age assessment is proposed to remove subtle human rater estimates of difference to achieve reported accuracy of 8.5 months with a precision of 2 months [5] and where sensitivity to image quality was reported to be less than 2.4 months [6]. Reports do, however, indicate that the computer based bone age systems find it difficult to automatically analyze variation, size, shape and mineralization in the target bones. Additional concern originates from the possible failure that comes from not having the radiograph reviewed by a trained clinician so that a possible defect originating from a clinical disorder is overseen.

For some time DXA technology has been offered as a technology that can be applied to execute a bone age assessment. DXA is widely available, produces studies swiftly and exposes the subject to very low radiation doses. Unlike the radiological methods which require time consuming changes both in content and structure, DXA relies on a directly quantitative assessment of mineral content. As such, DXA potentially provides a means to reflect even short term increases in mineral that have not yet been able to produce structural changes. Absent the need to identify subtle differences in structure has made DXA bone age assessments repeatable, accurate and largely independent of high skills $[2,7]$. As with the earlier study by Braillon and associates which finds a bone age assessment precision of $0.7 \%$ which- depending on age range-translates to either \pm 1 or \pm 2 months in bone age, we find precision to be in the range of $1.35 \%$ which translates to about 2.2 months in bone age. Similarly, when Heppe and associates compare bone age as assessed from the traditional radiographic bone age study and DXA they find a difference of 1.32 months with a Limit of Agreement between -9.84 months and 12.6 months which compares well with our finding a difference of -1.34 months and Limit of Agreement of -12.32 months and 9.64 months.

With the relatively low radiation dose, ease of performance and repeatability this DXA-based bone age assessment promises to be a useful reflector of short term change in bone age. Sensitivity to short term change was documented by comparing two bone age studies done over three months in 33 healthy boys and 34 healthy girls. That over a short three month period, 17 boys and 16 girls showed enough change to register a significant difference between studies documents a truly useful function delivered by DXA.

We suggest that the study confirms the DXA-based bone age assessment can be performed with precision and ease by routine staff. We do, however, find in studies comparing bone age by DXA and a traditional radiographic study that the DXA-based bone age study is not necessarily superior to the traditional radiographic study. Examining change with time, however, we do see that the DXA-based bone age study is especially sensitive to short-term change and we suggest that perhaps this is a uniquely valuable role for the DXA-based bone 
age study. We suggest that the best role for the DXAbased bone age study is to complement the traditional radiographic bone age assessment with a study to assess short term change in patients in a clinical setting.

\section{Sources of Support}

Self supported from efforts by the three authors.

\section{Author's Contribution}

All authors contributed to the study in collection of the data, analysis of the studies and producing of this report.

\section{References}

1. De Sanctis V, Di Maio S, Soliman AT, Giuseppe Raiola, Rania Elalaily, et al. (2014) Hand x-ray in pediatric endocrinology: Skeletal age assessment and beyond. Indian $\mathrm{J}$ Endocrinol Metab 18: S63-S71.

2. Braillon $P M$, Guibal $A L$, Pracos-Deffrenne $P$, Serban $A$, Pracros JP, et al. (1998) Dual energy x-ray absorptiometry of the hand and wrist-a possible technique to assess skeletal maturation: Methodology and data in normal youths. Acta Paediatr 87: 924-929.

3. Wang JM, Guo FW, Luo DM, Mei JH, Sanchez TV (2003) Multicenter study of carpal bone density measurement by Norland DXA scanners to assess skeletal maturation in Chinese boys and girls. J Bone Mineral Res. 18(S1): S387, 2003.

4. Martin DD, Wit JM, Hochberg Z, SavendahI L, Van Rijn RR, et al. (2011) The use of bone age in clinical practice-Part 1. Horm Res Paediatr 76: 1-9.

5. Thodberg $\mathrm{HH}$ (2009) An automated method for determination of bone age. J Clin Endocrinol Metab 94: 2239-2244.

6. Giordano D, Spampinato C, Scarciofalo G, Leonardi R (2010) An automatic system for skeletal bone age measurement by robust processing of carpal and epiphysial/ metaphysial bones. IEEE Transactions on Instrumentation and Measurement 59: 2539-2553.

7. Heppe DH, Taal HR, Ernst GD, Van Den Akker EL, Lequin MM, et al. (2012) Bone age assessment by dual-energy $\mathrm{x}$-ray absorptiometry in children: An alternative for x-ray? Br J Radiol 85: 114-120. 